\title{
Embarazo y parto vaginal en primigesta posterior a reconstrucción mamaria con colgajo TRAM*
}

\author{
Pregnancy and vaginal delivery after transverse rectus \\ abdominis musculocutaneous flap for breast reconstruction. \\ Report of one case
}

\author{
Drs. PATRICIO STEVENS M.1,2, RODRIGO VILLAGRÁN M. ${ }^{1,2}$, ALEJANDRA KLENNER M.1,2 \\ ${ }^{1}$ Servicio de Cirugía Hospital Las Higueras de Talcahuano. ${ }^{2}$ Departamento de Cirugía. Facultad de Medicina. \\ Universidad de Concepción. Octava Región, Chile
}

\begin{abstract}
RESUMEN
Introducción: La reconstrucción mamaria con colgajo miocutáneo transverso de recto anterior del abdomen (TRAM), es un recurso disponible en la rehabilitación del cáncer mamario. Existe poca literatura respecto de los cambios que experimenta la pared abdominal previamente debilitada con una gestación. Nuestro objetivo es divulgar nuestra experiencia en una paciente nuligesta, reconstruida con colgajo TRAM y que presentó embarazo de término por parto vaginal, sin incidentes. Material y Método: Análisis retrospectivo de ficha clínica. Paciente de 29 años sometida a radioterapia preoperatoria y mastectomía derecha simple extendida por cáncer mamario. Se realizó reconstrucción mamaria al año, con colgajo TRAM; se utilizó músculo recto unilateral, sin malla protésica, a los tres meses se reconstruyó pezón con injerto de piel perineal con técnica Skate-Flap. Se embarazó sin planificación en el postoperatorio inmediato de la reconstrucción de pezón. Inicia controles en unidad de alto riesgo obstétrico al quinto mes de gestación. Desarrolló un embarazo sin complicaciones, teniendo un parto vaginal de término con recién nacido masculino vivo de 2720 grs. El último control se realizó a los seis años, libre de enfermedad y no encontrándose hernia de la pared abdominal. Conclusiones: Los cambios que experimentó el abdomen, durante la gestación no afectaron la pared debilitada por la ausencia de uno de los músculos rectos abdominales. Se cumplió el objetivo de la reconstrucción mamaria respecto de recuperar la feminidad alterada por una mastectomía. El caso advierte al equipo médico respecto de tomar las precauciones ante la fertilidad en una mujer sexualmente activa.
\end{abstract}

\section{PALABRAS CLAVE: TRAM, embarazo, reconstrucción mamaria.}

\begin{abstract}
Mammary reconstruction using Transverse Rectus Abdominis Musculocutaneos Flap (TRAM) is successfully used for mammary reconstruction after breast cancer surgery. The absence of the anterior rectus muscle modifies the functional properties of the abdominal wall. There is little knowledge about what occurs with a debilitated abdominal wall during pregnancy after mammary reconstruction using a TRAM flap. We report a 29 years old patient, who was treated for breast cancer with preoperative radiotherapy and a right side mastectomy with lymphadenectomy. TRAM flap was done one year later; a single rectus
\end{abstract}

*Recibido el 14 de Octubre de 2007 y aceptado para publicación el 9 de Noviembre de 2007.

Correspondencia: Dr. Patricio Stevens M.

Aníbal Pinto 215. Oficina 502. Concepción, Chile

e-mail:pstevens@udec.cl 
abdominis was used and the abdominal wall was not reinforced with a mesh. After three months, the nipple was reconstructed with the skate flap technique. Although it was not planned, she got pregnant and did not search for obstetric assessment until the fifth month. She was followed at the high risk obstetric unit and no complications were observed in her abdominal wall. She had a vaginal delivery of a healthy full term male newborn weighing 2720 grams. Six years later, there was no evidence of abdominal wall complications. Therefore, abdominal stress during pregnancy did not affect the debilitated abdominal wall after rotation of the rectus abdominis muscle.

\section{KEY WORDS: TRAM flap, pregnancy, mammary reconstruction.}

\section{INTRODUCCIÓN}

La reconstrucción con Colgajo TRAM ha sido utilizada con éxito para la reconstrucción mamaria desde hace ya mas de 20 años ${ }^{1,2}$. Siendo de gran importancia en el tratamiento integral y completo de la patología neoplásica mamaria, especialmente desde el punto de vista psicológico y realización de la feminidad de estas pacientes ${ }^{3}$.

Mucho se ha descrito respecto a la efectividad del colgajo TRAM y sus buenos resultados; sin embargo sólo casos aislados se encuentran en la literatura, respecto de la capacidad de la pared abdominal luego de la reconstrucción para soportar un embarazo de término y un parto vaginal.

Se ha escrito sobre la contraindicación del embarazo luego de la reconstrucción con Colgajo TRAM posterior a una mastectomía por cáncer

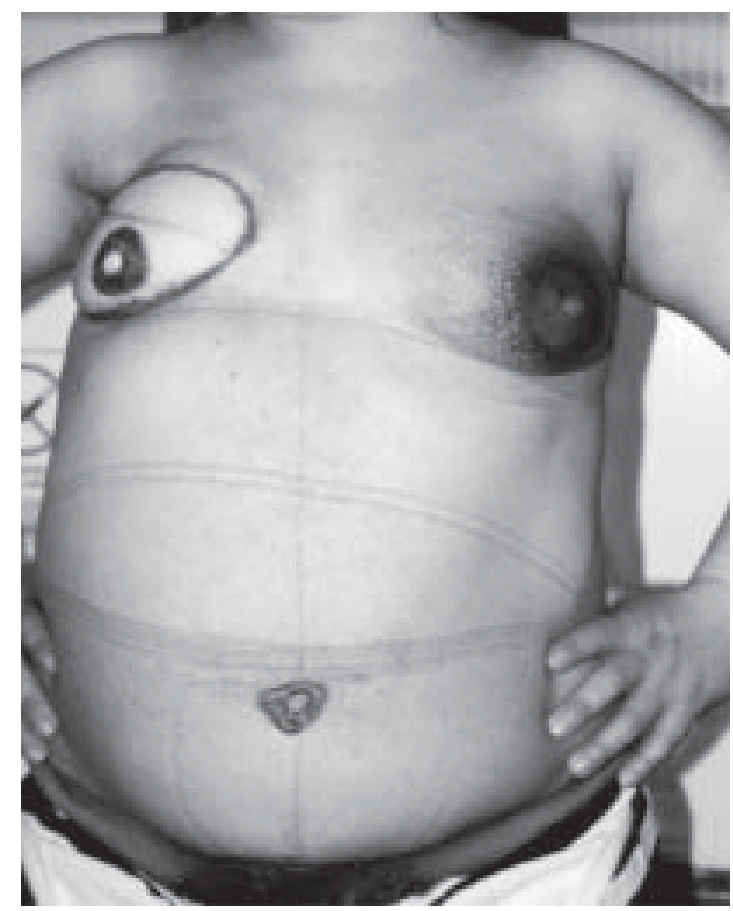

Figura 1. Reconstrucción con colgajo TRAM de mama derecha. 25 semanas de embarazo, vista anterior. mamario ${ }^{4}$, mas sólo son aseveraciones sin fundamentos.

El diagnóstico cada vez más temprano y el aumento sustancial de la sobrevida en pacientes en edad fértil se traducirán en que veamos cada vez con mayor frecuencia este tipo de casos ${ }^{5}$. Esto último es de vital importancia a la hora de enfrentar las dudas de estas pacientes con respecto a su paridad.

\section{CASO CLÍNICO}

Paciente sin antecedentes mórbidos de importancia, consulta a la edad de 29 años por historia de 2 años de dolor mamario derecho y masa dura retroareolar, asociado a retracción y secreción purulenta intermitente por pezón ipsilateral, durante los últimos 9 meses.

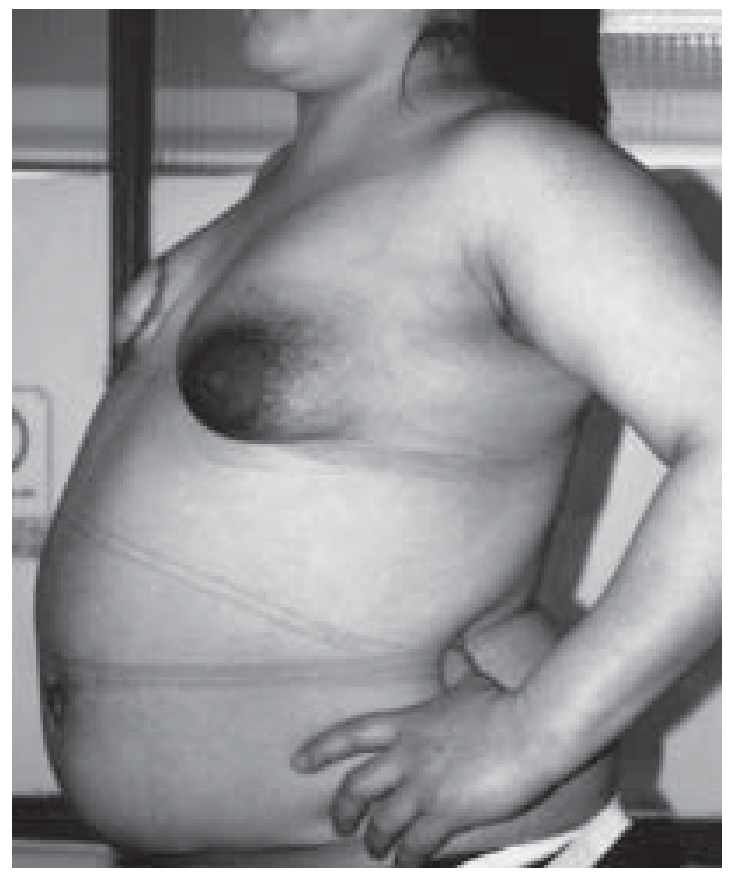

Figura 2. Reconstrucción con colgajo TRAM de mama derecha. 25 semanas de embarazo, vista lateral izquierda. 


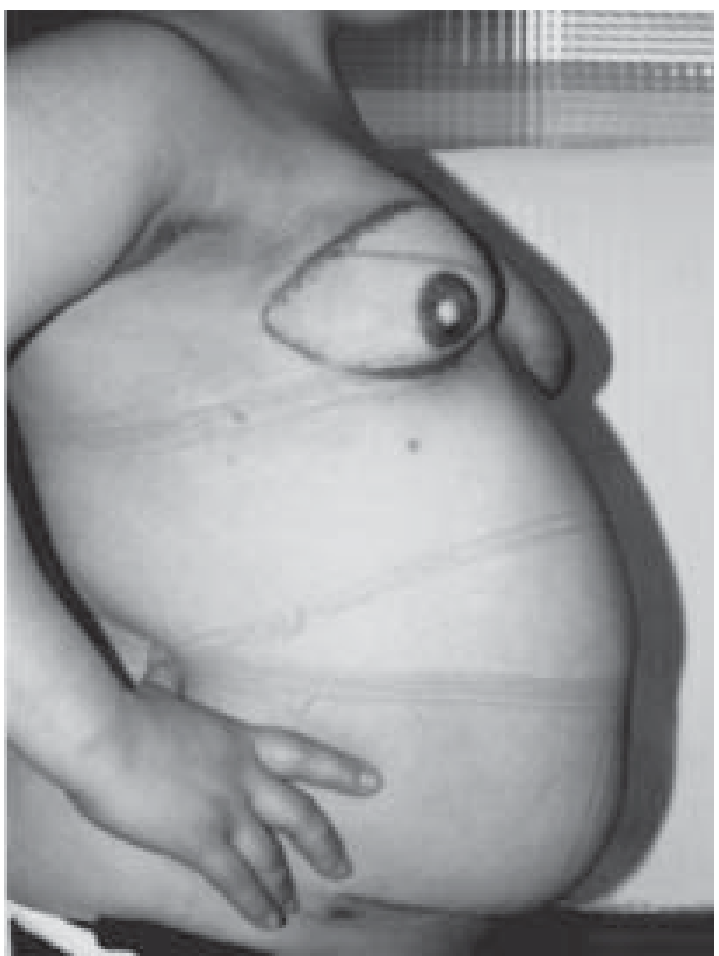

Figura 3. Reconstrucción con colgajo TRAM de mama derecha. 25 semanas de embarazo, vista lateral derecha.

Al examen físico se describe pezón derecho deformado y retraído. Masa retroareolar dura, irregular, adherida al pezon de 2,5×4 cm diámetro. Adenopatías axilares palpables ipsilaterales Mama contralateral sin lesiones.

La Mamografía evidencia pezón retraído, piel areolar engrosada, área isodensa retroareolar de 4 x $4,5 \mathrm{~cm}$ de límites imprecisos con distorsión de la

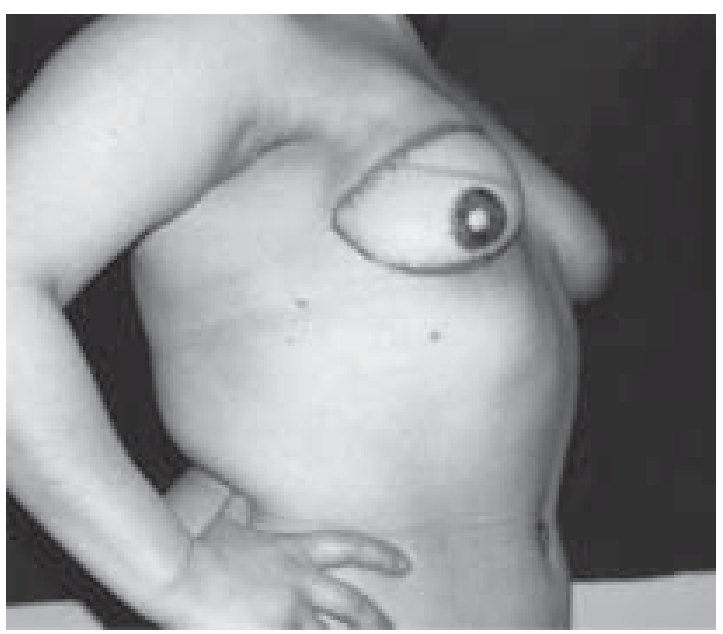

Figura 4. (a) 5 meses postembarazo, vista lateral derecha. (b) 5 meses postembarazo, vista antero-lateral izquierda. No se aprecian hernias ni eventraciones abdominales. arquitectura glandular, en contacto con complejo areola-pezón, con microcalcificaciones. Punción biopsia informa Adenocarcinoma, sugiriendo Enfermedad de Paget. El estudio de diseminación resultó negativo.

Se realiza radioterapia y posterior Mastectomía derecha simple y linfadenectomía axilar.

La biopsia confirma carcinoma ductal invasor. Tipo histológico: ductal NOS. Componente ductal in situ y permeación vascular positivos, bordes quirúrgicos y ganglios linfáticos negativos.

Un año más tarde se realiza reconstrucción mamaria con colgajo TRAM contralateral monopediculado, sin refuerzo protésico, con buena evolución postoperatoria, sin complicaciones. Tres meses más tarde se realiza reconstrucción del complejo areola-pezón con injerto de piel total de zona perineal con técnica Skate-flap, con buena evolución.

Un año después de la reconstrucción presenta embarazo no planeado con primera evaluación a los 5 meses de gestación, siendo controlada postefinalizando a las 38 semanas por parto vaginal espontáneo sin incidentes y recién nacido vivo de $2720 \mathrm{gr}$, con examen de pared abdominal sin alteraciones. Se controla periódicamente, con un último control a los 9 años 4 meses postparto, encontrándose paciente en muy buenas condiciones generales y emocionalmente muy satisfecha.

\section{DISCUSIÓN}

Los cambios de la pared abdominal, luego de la reconstrucción con Colgajo Miocutáneo de Músculo Recto Abdominal han sido cuidadosamente estudiados desde los inicios de la técnica des-

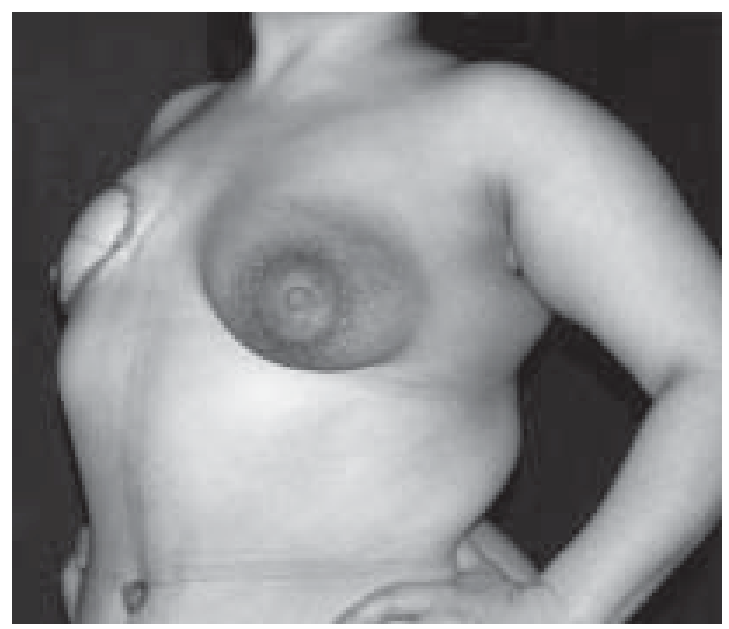
riormente en la Unidad de Alto Riesgo Obstétrico, 


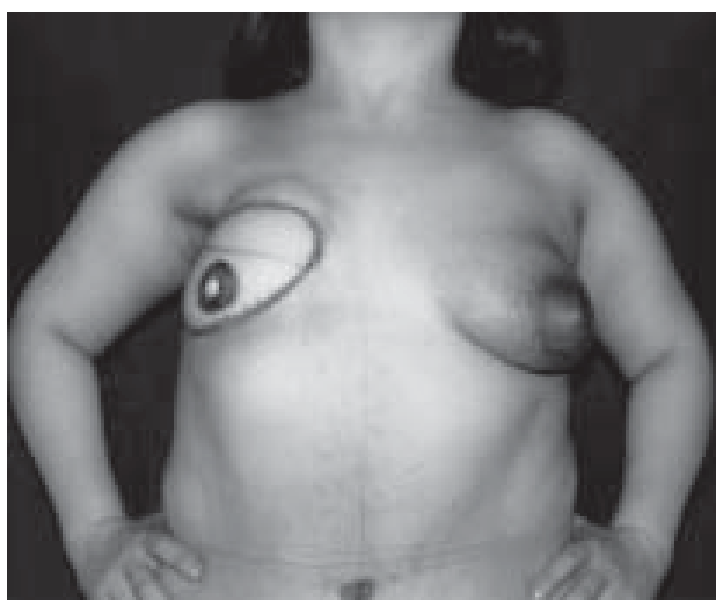

Figura 5. Cinco meses postembarazo, vista anterior.

crita por Drever y Robbins en 1977 y 1979 en forma independiente ${ }^{5-8}$, Sin embargo, no ha sido encontrada una solución única para las complicaciones. La debilidad de la pared abdominal, secundaria a la rotación del músculo que irriga el colgajo es responsable de una serie de problemas funcionales y estéticos que en los últimos años han despertado mayor interés ${ }^{5}$.

Pocos casos de embarazo exitoso, posterior a reconstrucción mamaria con Colgajo TRAM se han publicado en la literatura $2,5,9-14$, así como también casos con complicaciones; sin embargo, estos últimos sólo se tratan de descripciones aisladas de casos clínicos. La serie más numerosa encontrada en la literatura es de Chen, publicando en 1993, 7 casos de embarazos exitosos, 6 de los cuales culminaron con parto vía vaginal; dentro de ésta en 4 pacientes se habrían utilizado inclusive, ambos músculos rectos abdominales ${ }^{12}$. No obstante, muy raros casos de complicaciones de la pared también se han descrito, tal es el caso de Parodi y cols. quiénes describen una herniación de importancia posteriormente al embarazo, requiriendo reparación quirúrgica de la pared ${ }^{5}$.

Mizgala y cols. en su extensa serie de 150 pacientes, reconstruidas con colgajo TRAM, de las cuales realizó seguimiento a 137 sobrevivientes, sostiene que no hay mayores problemas referente a la realización de ejercicios físicos y reporta en su publicación sólo unos pocos casos de herniación y abombamiento de la pared abdominal $(2-6 \%)^{13}$. No obstante otros autores han contraindicado el embarazo luego de este tipo de reconstrucción ${ }^{15}$.

En nuestro caso fue posible devolver la feminidad a nuestra paciente, luego de la mastectomía y posterior reconstrucción con colgajo TRAM, incluso logra un embarazo fisiológico de término con parto vaginal.

Basados en nuestro caso y apoyados en la literatura, aunque escasa, creemos que no debemos contraindicar el embarazo luego de la reconstrucción con colgajo TRAM. Sin embargo debemos advertir claramente de las posibles complicaciones a las que se puede ver enfrentada durante su embarazo y parto; de modo de tomar las precauciones necesarias y proporcionar orientación adecuada en cada caso.

\section{REFERENCIAS}

1. Moore, HC, Foster RS Jr. Breast cancer and pregnancy. Semin Oncol 2000; 27: 646.

2. Johnson M. Vaginal Delivery of Monozygotic Twins after Bilateral Pedicle TRAM Breast Reconstruction. Plastic \& Reconstructive Surgery 2002; 109:16531654.

3. Wagner LH. Pregnancy after a TRAM flap procedure: principles of nursing care. J Obstet Gynecol Neonatal Nurs 2000; 29: 363-368.

4. Upponi SS. Pregnancy after Breast Cancer. European Journal of Cancer 2003; 39: 736-741.

5. Parodi PC. Pregnancy and Tram-Flap Breast Reconstruction after Mastectomy: A Case Report. Scand J Plast Reconstr Hand Surg 2001; 35: 211215.

6. Drever JM. The Epigastric Island Flap. Plastic \& Reconstructive Surgery 1977; 59: 343-346.

7. Robbins TH. Rectus abdominis myocutaneous flap for breast reconstruction. Aust N Z J Surg 1979; 49: 527-530.

8. Robbins TH. Post-mastectomy breast reconstruction using a rectus abdominis musculocutaneous island flap. Br J Plast Surg 1981; 34: 286-290.

9. Kroll S. Abdominal Wall Strength, Bulging, and Hernia after TRAM Flap Breast Reconstruction. Plastic \& Reconstructive Surgery 1995; 96: 616-619.

10. Menz P. Pregnancy after Abdominoplasty. Plastic \& Reconstructive Surgery 1996; 98 :377-378.

11. Borman $H$. Pregnancy in the early period after abdominoplasty. Plast Reconstr Surg 2002; 109: 396-397.

12. Chen L. Successful pregnancies following TRAM flap surgery. Plast Reconstr Surg 1993; 91: 69-71.

13. Mizgala CL. Assessment of the abdominal wall after pedicled TRAM flap surgery: 5- to 7-year follow-up of 150 consecutive patients. Plast Reconstr Surg 1994; 93: 988-1002.

14. Viterbo, F. Pregnancy after TRAM flap. Plast Reconstr Surg 1993; 92: 1198.

15. Sakai S, Takahashi $H$, Tanabe $H$. The extended vertical rectus abdominis myocutaneous flap for breast reconstruction. Plast Reconstr Surg 1989; 83: 1061-1067. 